\title{
Analyse génétique des étapes précoces de la progression tumorale
}

Le développement des tumeurs est un processus séquentiel qui peut être analysé expérimentalement : on a ainsi identifié dans des systèmes expérimentaux modèles, différentes classes d'oncogènes qui agissent en synergie pour aboutir à la transformation de la cellule. Une étape ainsi définie correspond à l'immortalisation, à partir de laquelle peuvent agir d'autres gènes et facteurs promoteurs.

Evelyne Mougneau

Assistante à l'Université de Nice.

\section{Nicolas Glaichenhaus}

Elève de l'École Normale Supérieure.

\section{François Cuzin \\ Professeur à l'Université de Nice.}

\section{REFERENCES}

r. Haaland M. Spontaneous tumors in mice. $S_{C i}$ Rep Imperial Cancer Res Fund, 1911; $4: 1$.

2. Medina D. In: Becker F F ed., Cancer, vol. 3, New York: Plenum Press, 1975; 99-I 19.

3. Stéhelin D, Varmus HE, Bishop JM, Vogt PK. DNA related to the transf orming gene (s) of avian sarcoma viruses is present in normal avian DNA. Nature 1976; $260: 170-3$.

4. Duesberg $\mathrm{PH}$. Retroviral transforming genes in normal cells? Nature 1983; $304: 219-26$.

5. Jariwalla RJ, Aurelian L, Ts'o PO. Immortalization and neoplastic transformation of normal diploid cells by defined cloned fragments of herpes simplex virus type 2. Proc Natl Acad Sci $U S A$ 1984; 80 : 5902-6.

\section{ADRESSE}

E. Mougneau, N. Glaichenhaus, F. Cuzin : Unité de Génétique Moléculaire des Papovavirus, (Inserm U 273 alliée au Cnrs), Centre de Biochimie de l'Université de Nice, Parc Valrose, 6034 Nice Cedex.

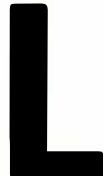

'existence d'étapes successives dans le développement des cancers fut reconnue dès le début du siècle : dès I9I I, Haaland [I] décrivait plusieurs étapes dans l'évolution du cancer du sein. Les travaux de Rous sur l'évolution des papillomes en carcinomes, ainsi que ceux de Foulds sur la dépendance hormonale des tumeurs mammaires [2], conduisirent à généraliser l'idée d'une progression des tumeurs par l'acquisition de nouvelles propriétés par des cellules initialement modifiées. Le caractère en général irréversible de cette progression suggérait qu'elle résultait de l'accumulation de mutations somatiques, probablement dans des gènes différents, étant donné la variété des altérations observées.

L'existence de gènes transformants (oncogènes) a été d'abord démontrée chez des virus à $\mathrm{ADN}$ (polyome) et à ARN (rétrovirus). Chez le prototype de ce dernier groupe, le virus du sarcome de Rous, l'expression d'un seul gène (src) permet au virus d'induire des sarcomes in vivo et de transformer in vitro des fibroblastes de poulet. A la suite des observations faites en 1976 par Stéhelin et al. [3], de nombreux travaux montraient que l'oncogène src était homologue d'un gène cellulaire de poule (protooncogène). Une situation a été décrite dans le cas d'autres rétrovirus qui comportent le plus souvent un, parfois deux, oncogènes homologues de gènes cellulaires [4]. Ces oncogènes viraux apparaissent donc comme des formes altérées de gènes cellulaires normaux. Parallèlement, les progrès des techniques de clonage et de transfert de gènes ont permis de commencer à rechercher des oncogènes dans des tumeurs d'origines diverses. Plusieurs auteurs ont ainsi montré que les cellules de différentes tumeurs contiennent dans leur génone des séquences d'ADN capables de transformer des fibroblastes de souris en culture.

Il apparaissait à ce stade encore difficile d'établir une liaison entre l'un des oncogènes connus, viraux ou cellulaires, et une étape définie d'un processus de transformation. C'est sur un autre système modèle, celui fourni par les virus oncogènes à $\mathrm{ADN}$, que put être soumise à l'expérience l'idée que différents oncogènes pourraient agir sur des cibles cellulaires distinctes à des étapes définies du processus tumoral.

\section{Oncogènes multiples des virus à $A D N$}

Contrairement à la plupart des rétrovirus, ces virus comportent plusieurs oncogènes qui agissent de concert pour établir l'état transformé. Deux cas ont été étudiés en détail, le virus polyome et les adénovirus. Des données préliminaires dans le même sens ont été 
rapportées récemment sur deux virus d'importance médicale, l'herpès simplex type 2 et le virus d'Epstein-Barr [5, 6].

Le virus polyome : trois protéines virales sont exprimées dans les lignées transformées de fibroblastes de rongeurs ( $f i g . I$ ) : la protéine grand-T, associée à la chromatine, la protéine moyen- $T$, associée aux membranes et la protéine petit- $T$, cytosolique. Le transfert dans des cellules en culture de génomes viraux modifiés [7] codant pour une seule des trois protéines (gènes plt, pmt et pst respectivement) a permis de préciser leurs rôles dans la transformation cellulaire [8-10]. La protéine moyen- $T$ est capable de transformer des cellules de lignées établies mais pas des fibroblastes embryonnaires en culture (cultures primaires). Dans ce dernier cas, le transfert successif des gènes plt et pmt permet d'établir des lignées transformées. Les cellules n'ayant reçu que le gène plt expriment seulement une partie des caractères de transformation : capacité de se diviser en culture pendant un nombre illimité de générations, diminution des exigences en facteurs sériques. Cependant, ces cellules conservent la morphologie de fibroblastes non transformés, comme on peut le voir en figure 2.
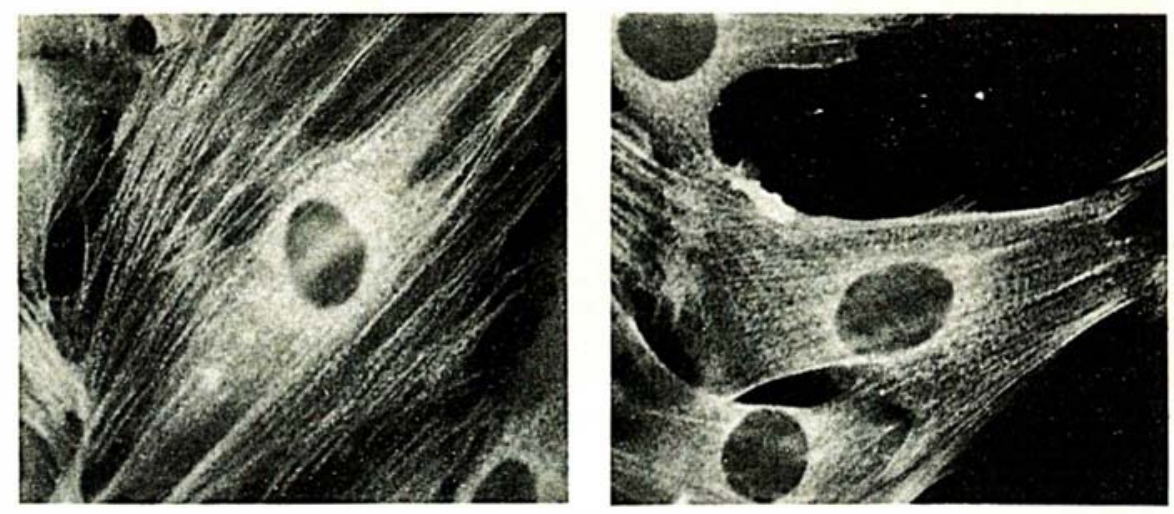

Figure 2. Morphologie de fibroblastes immortalisés par la protéine grand-T ou transformés par le virus polyome.

Les cellules en culture sont marquées par un anticorps dirigé contre une protéine du cytosquelette (actine) et couplé à un anticorps fluorescent :

A: fibroblastes embryonnaires de rat en culture primaire.

B: fibroblastes de rat immortalisés par la proteine grand $-T$ du virus polyome.

C: fibroblastes de rat transformés par le virus polyome et exprimant les trois protéines précoces.

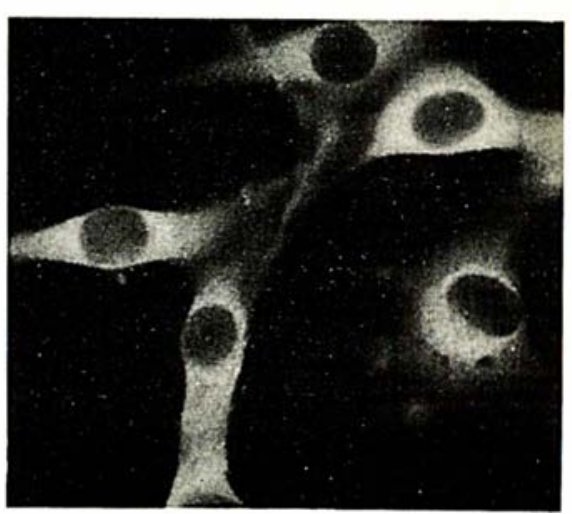

Figure 1. Carte physique de la région précoce du génome du virus polyome.

Trois ARN messagers ( $\square$ ) correspondant aux trois protéines précoces sont synthétisés grâce aux trois épissages différents (- $\longrightarrow$ ) d'un même $A R N$ précurseur. Les trois cadres de lecture $[1,2,3]$ sont également indiqués.

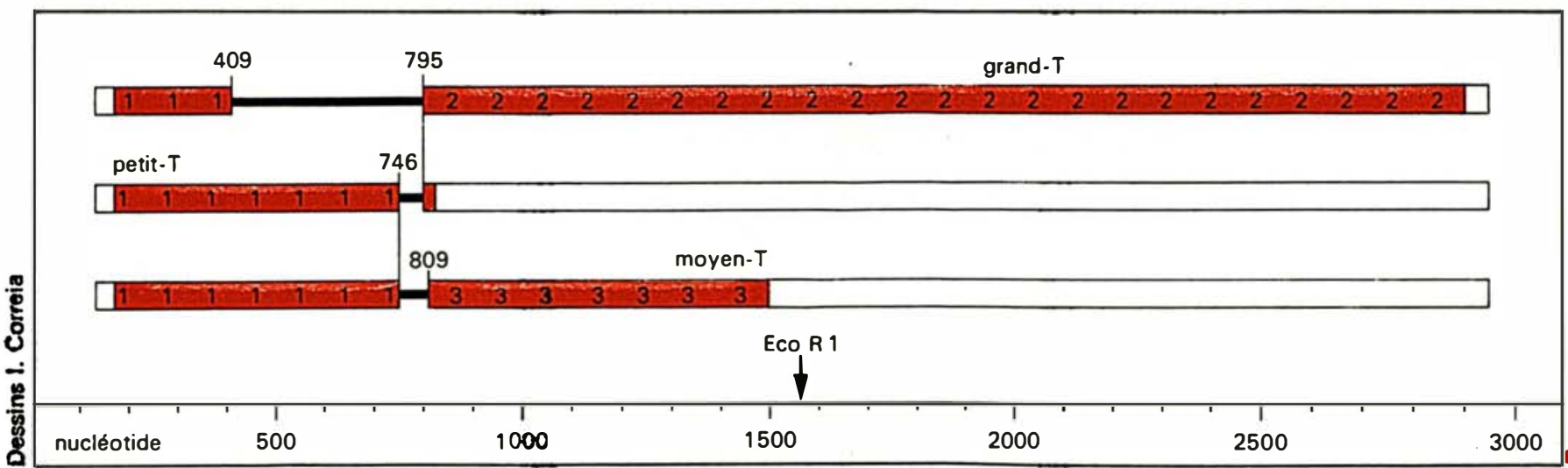




\section{REFERENCES}

6. Griffin BE, Karran L. Immortalization of monkey epithelial cells by specific fragments of Epstein-Barr virus DNA. Nature 1984; 309: 7882.

7. Treisman $\mathbf{R}$, Novak U, Favaloro J, Kamen $\mathbf{R}$. Transformation of rat cells by an altered polyoma virus genome expressing only the middle- $T$ protein. Nature 1981; $292:$ 595-600.

8. Rassoulzadegan M, Cowie A, Carr A Glaichenhaus $\mathbf{N}$, Kamen $R$. The roles of individual polyoma virus early proteins in oncogenic transformation. Nature 1982; $300: 713-8$.

9. Rassoulzadegan $M$, Naghashfar Z, Cowie A, Cuzin F, et al. Expression of the large $T$ protein of polyoma virus promotes the establishment in culture of "normal " rodent fibroblast cells. Proc Natl Acad Sci USA 1983; $80: 4354-8$.

10. Cuzin F. The polyoma virus oncogenes: coordinated functions of three distinct proteins in the transformation of rodent cells in culture. Biochim Biophys Acta 1984; 781 : 193-204.

II. Houweling A, Van den Elsen P J, Van der Eb AJ. Partial transformation of primary rat cells by the leftmost $4.5 \%$ fragment of adenovirus 5 DNA. Virology 1980; $105: 537-50$.

12. Van der Elsen PJ, de Pater S, Houweling A, Van der Veer J, Van der Eb A. The relationship between region $E_{1} A$ and $E_{1} B$ of human adenoviruses in cell transformation. Gene 1982; $18: 175-85$.

13. Land H, Parada LF, Weinberg RA. Tumorigenic conversion of primary embryo fibroblasts requires at least two cooperating oncogenes. Nature 1983; $304:$ 596-602.

14. Ruley HE. Adenovirus early region IA enables viral and cellular transforming genes to transform primary cells in culture. Nature 1983; 304: 602-6.

15. Murray MJ, Cunningham JM, Parada LF Dautry F, Lebowitz P, Weinberg RA. The HL60 transforming sequence : a ras oncogene coexisting with altered myc genes in hematopoietic tumors. Cell 1983; $33: 749-57$.

16. Mougneau E, Lemieux L, Rassoulzadegan M, Cuzin F. Biological activities of the $v$-myc and of rearranged $c$-myc oncogenes in rat fibroblast cells in culture. Proc Natl Acad Sci USA 1984; $81: 575^{8-62}$.

17. Ruley HE, Moomaw JF, Maruyama K Avian myelocytomatosis virus myc and adenovirus early region $\mathrm{E}_{\mathrm{I}} \mathrm{A}$ promote the in vitro establishment of cultured primary cells. Cancer Cells, Cold Spring Harbor 1984; 2 : 481-6.

18. Müller R, Verma IM. Expression of cellular oncogenes. Current Topics Microbiol Immunol 1984; 112 : 73-115.

19. Graf T, Beug H. Avian leukemia viruses: interactions with their arget cells in vivo and in vitro. Biochim Biophys Acta 1978; $516: 269-99$
Adénovirus : les cellules transformées par les adénovirus ne contiennent généralement qu'une partie du génome viral, correspondant à certains gènes précoces (région $E_{I}, f i g$. 3), dont le transfert est suffisant pour conduire à la transformation complète. Deux unités de transcription, $E_{I} A$ et $E_{I} B$ y ont été identifiées; aucune n'est suffisante pour induire seule la transformation de fibroblastes embryonnaires de rat ou de hamster en culture. Le transfert de la région $E_{I} A$ permet, comme celui du gène plt , l'établissement de lignées non tumorigènes; par contre, le transfert de la seule région $E_{I} B$ ne produit aucun effet visible. Ces résultats indiquent l'existence de deux étapes avec un état intermédiaire correspondant à des fibroblastes non tumorigènes immortalisés [I I, I2].

\section{Coopération entre oncogènes cellulaires}

Un phénomène de coopération entre plusieurs gènes pour l'établissement du phénotype transformé, comparable à celui qui avait été découvert chez les adénovirus et polyomavirus, a été décrit dans le cas d'oncogènes cellulaires par Land et al. [13] et par Ruley [14]. Les oncogènes du groupe ras sont capables de transformer les cellules d'une lignée établie de fibroblastes de souris $\left(\mathrm{NIH}_{3} \mathrm{~T}_{3}\right)$ mais pas de transformer des fibroblastes embryonnaires de rongeur. D'autre part, l'existence dans des cellules de lignées tumorales d'au moins deux oncogènes activés ( $m y c$ et ras) [ 15 ] suggérait que l'oncogène $m y c$ pou- vaient coopérer avec l'oncogène ras. De fait, le transfert simultané des deux oncogènes dans des cellules embryonnaires permettait d'obtenir des cellules tumorigènes dans la souris nude [13]. De plus, $m y c$ pouvait être remplacé par les gènes plt ou $E_{I} A$, ou encore par la forme virale v-myc du rétrovirus MC29. De ces expériences, les auteurs ont conclu à l'existence de deux classes d'oncogènes, chacun des gènes du groupe I $\left(m y c, p l t, E_{I} A\right)$ pouvant coopérer avec l'un des gènes du groupe II (ras, pmt, $E_{I} B$ ) pour la transformation de cellules embryonnaires. Le transfert de l'un des gènes du groupe I, insuffisant pour établir une transformation complète, conférait à la cellule la capacité de se multiplier indéfiniment en culture (" immortalisation"). Ceci avait été montré pour les gènes plt et $E_{I} A$ et pouvait effectivement être étendu aux formes activées de $m y c$ $[16,17]$.

L'une des modifications qui peuvent aboutir à l'activation d'un proto-oncogène cellulaire est son expression à un taux accru, sans changement de structure de la protéine produit du gène [18]. Il apparaît donc que pour certains gènes ( $m y c$, mos, ras, fos), la surexpression de la protéine normale a un effet comparable à celui d'une mutation dans la région codante. Ce phénomène, analogue aux " eff ets de dose " de la génétique classique, se retrouve dans l'analyse plus fine du rôle de ces gènes' dans les étapes précoces de la transformation.

Ainsi, la classification du gène v-myc parmi les gènes du groupe I pouvait être considérée comme

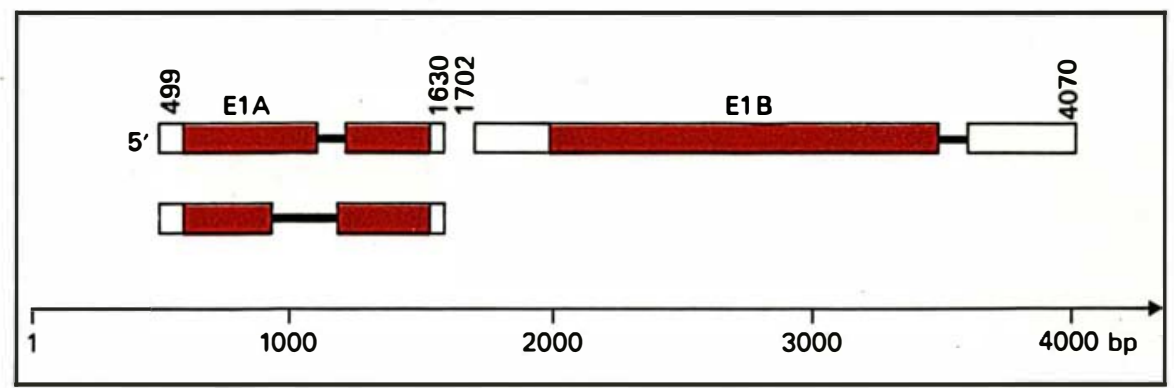

Figure 3. Carte physique de la région codant pour les messagers $E_{I} A$ et $E_{I} B$ de l'adénovirus (d'après la référence [12]). bp=paires de bases.

Les messagers sont représentés par des rectangles, blancs pour la partie non codante, rouges pour la partie codante. Les introns sont représentés par des traits pleins. Voir aussi notre Lexique dans Médecine-Sciences I, page 48. 
paradoxale. De fait ce gène n'est capable de transformer, après transfection, ni des cellules de lignées établies, ni des cellules embryonnaires de rongeurs et ces dernières sont immortalisées [ $\mathrm{I}_{3}$, I6, 17]. Cependant, l'infection de cellules de poulet par le virus MC29, porteur de ce même gène, conduit à une transformation complète [19]. De plus, Vennström et al. [20] ont rapporté récemment que le génome d'un rétrovirus murin, construit in vitro et contenant le gène $m y c$ du virus $\mathrm{OK}_{\mathrm{I}}$, était capable de transformer des fibroblastes de souris ou en culture primaire. Ces propriétés différentes sont vraisemblablement liées à des niveaux d'expression différents du gène. L'importance de ces effets de dose a également été mise en évidence par Spandidos et Wilkie [2I] dans le cas du gène ras. Bien que ne remettant pas en cause le principe d'un déterminisme génétique d'étapes définies dans la transformation de fibroblastes embryonnaires, ces résultats soulignent le caractère nécessairement partiel de tels modèles pour rendre compte d'un phénomène biologique complexe.

\section{Immortalisation en culture et cancer}

Les gènes du groupe I sont capables par eux-mêmes d'altérer de manière significative les propriétés de croissance de cellules en culture $[8,9,16$, I7]. La modification la plus spectaculaire est l'acquisition, par des fibroblastes embryonnaires, des deux propriétés caractéristiques de l'immortalité clonale en culture [22] : la capacité de former des colonies après ensemencement à faible densité et la croissance en culture pour un nombre de générations apparemment illimité. De plus, ces cellules deviennent capables de se diviser dans des milieux ne contenant que de faibles concentrations en facteurs sériques et peuvent être transformées par des promoteurs chimiques.

Une question souvent posée est celle des rapports possibles entre I'acquisition du phénotype immortalisé opérationnellement défini en culture de cellules et une étape de la progression tumorale in vivo.

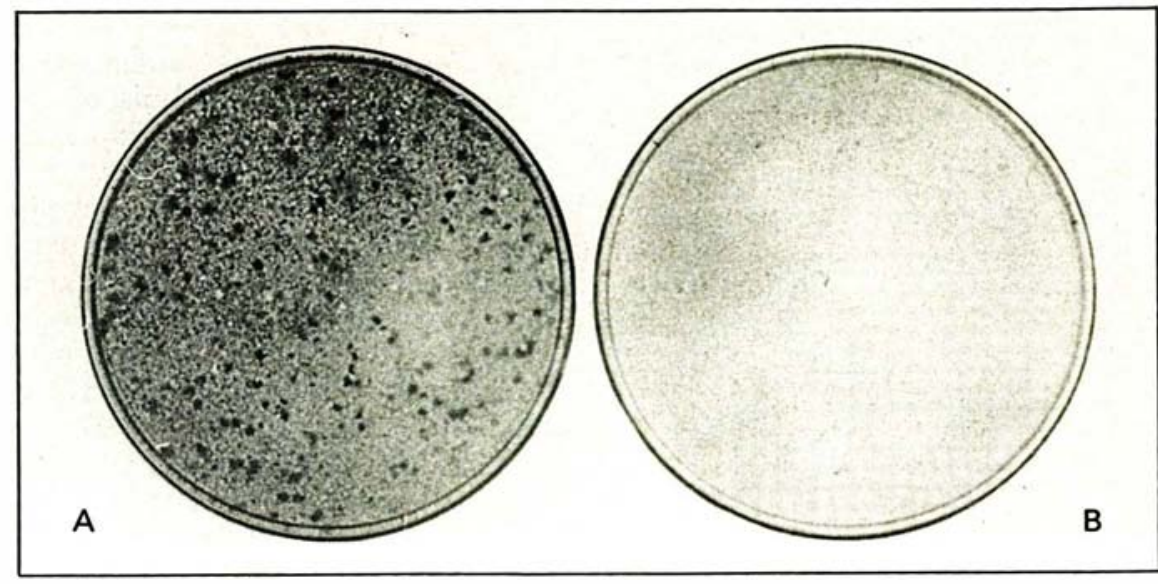

Figure 4. Fibroblastes de rat exprimant la protéine grand-T traités $(A)$ ou non $(B)$ au TPA.

Après traitement au TPA, des foyers de cellules transformées apparaissent sur la monocouche cellulaire.

Quoique " l'immortalisation " des fibroblastes de rongeur soit en effet analysée dans des conditions de croissance tout à fait artificielles, plusieurs arguments expérimentaux indiquent cependant que cette " immortalité " ne correspond pas seulement à un artefact imposé par l'expérimentation in vitro [13, 23].

Les propriétés particulières conférées par les oncogènes du groupe I indiquent qu'il est possible de rechercher de nouveaux oncogènes de ce type qui seraient activés au cours d'étapes précoces de la transformation maligne. La stratégie, analogue à celle qui avait amené à l'isolement des oncogènes de la famille ras, consisterait à transférer l'ADN de cellules tumorales dans des cellules $3 \mathrm{~T}_{3}$ et à sélectionner, non pas pour l'expression du phénotype transformé (formation de foyers), mais pour un des caractères spécifiques du groupe I. L'un de ceux-ci est l'indépendance des facteurs sériques pour la croissance en culture (croissance en milieu pauvre en sérum $[8, \mathrm{I} 6]$ ou en plasma [24]). Une autre sélection possible est fondée sur l'observation que les cellules exprimant ces gènes réagissent non seulement à l'expression d'oncogènes activés du groupe II, mais aussi à des cancérogènes chimiques. Les cellules exprimant les protéines du groupe I ne sont pas seulement sensibles à l'effet transformant des oncogènes du groupe II, elles deviennent également capables d'exprimer un phénotype transformé après traitement par des promoteurs chimiques tel que le i2-Otetradecanoyl - phorbol - I3 - acétate (TPA) [25] (fig. 4). Ces composés promoteurs avaient été identifiés lors d'études sur la cancérogénèse chimio-induite de cellules de peau [26] : ils confèrent, les caractères de transformation terminale à des cellules qui ont déjà subi un événement dit d'initiation, et non à des cellules normales. Le parallélisme est frappant entre ces cellules "initiées" et celles exprimant les gènes plt ou myc, qui sont transformées par un traitement pour un temps limité par le TPA. Cette observation, utile sur le plan expérimental pour la recherche de nouveaux oncogènes de ce groupe, présente l'intérêt conceptuel de caractériser l'état "immortel " induit par ces gènes comme un état "à haut risque ": la cellule, bien que normale pour la plupart de ses propriétés, est engagée dans une voie conduisant à un état tumoral et devient réactive vis-à-vis de stimuli qui sont sans effet sur une cellule vraiment normale.

\section{Étapes précoces de la transformation}

Les résultats expérimentaux résumés dans cette revue permettent de définir une étape précoce de la transformation tumorale dépendant de l'expression des oncogènes plt, 


\section{REFERENCES}

20. Vennström B, Kahn P, Adkins B, et al. Transformation of mammalian fibroblasts and macrophages in vitro by a murine retrovirus encoding an avian $v$-myc oncogene. The EMBO $\mathrm{J}$ 1984; 3 .

21. Spandidos DA, Wilkie NM. Malignant transformation of early passage rodent cells by a single mutated human oncogene. Nature 1984; 310: 469-75.

22. Todaro GJ, Green H. Quantitative studies on the growth of mouse embryo cells in culture and their devclopment into established lines. I Cell Biol 1963; $17: 299-313$.

23. Assclin C, Gélinas C, Bastin M. Role of the three Polyoma virus carly proteins in tumorigenesis. Mol Cell Biol 1983; 3 : 1451-7.

24. Armclin HA, Armelin MCS, Kelly K, Functional role for $c$-myc in mitogenic response to platelet-derived growth factor. Nature 1984; 311 : 655-60.

25 Connan G, Rassoulzadegan M, Cuzin F. Focus formation in rat fibroblasts exposed to a tumor promoter after transfer of polyoma plt and myc oncogenes. Nature 1985 ; sous presse.

26. Heidelberger C. Chemical carcinogenesis. Annu Rev Biochem 1975; 44 : 79-111.

27. Orth G, Favre M, Breitburd F, et al. Epidermodysplasia verruciformis: a model for the role of papilloma-viruses in human cancer. Cold Spring Harbor Conference on Cell Proliferation 1980; $7: 259-82$.

28. Boshart M, Gissmann L, Ikenberg H, Kleinheinz A, Sheurlen W, Zur Hausen $H$. A new type of papillomavirus DNA, its presence in genital cancer biopsies and in cell lines derived from cervical cancer. The $E M B O$ J 1984; $3: 1151-7$.

29. Grisoni M, Meneguzzi G, De Lapeyric̀re $O$, Binétuy B, Rassoulzadegan M, Cuzin F, et al. The transformed phenotype in culture and tumorigenicity of Fischer rat fibroblast cells $\left(\mathrm{FR}_{3} \mathrm{~T}_{3}\right)$ transformed with bovine papilloma virus type Virology 1984; $135: 406-16$.

30. Meneguzzi G, Binétruy B, Grisoni M, Cuzin F. Plasmidial maintenance in rodent fibroblasts of a $B P V_{1}-\mathrm{pBR}_{322}$ shuttle vector without immediately apparent oncogenic transformation of the recipient cells. The EMBO 3 1984; $3: 365-71$.
$E_{I} A$ et $m y c$. L'expression de ces gènes ne conduit pas seulement à l'expression par des fibroblastes de rongeur de nouvelles propriétés comme l'immortalisation et l'indépendance en sérum, elle crée également un état de " haut risque " dans lequel ces cellules deviennent capables d'acquérir un phénotype complètement transformé en réponse à des stimuli extérieurs. Il est clair que l'étude approfondie de ces étapes précoces de cancérisation sera essentielle pour comprendre un jour l'origine d'une évolution néoplasique et pour mettre au point de nouvelles thérapeutiques.

Une question importante est celle de la généralité de l'étape précoce définie par les oncogènes du groupe I. Une vision optimiste (sans doute à l'excès) serait que tous les cancers commencent par une étape similaire à celle décrite après transfert d'un gène myc activé dans le fibroblaste de rongeur, avec seulement quelques variations selon le tissu ou l'espèce. L'hypothèse pessimiste serait que l'immortalisation de cellules de rongeurs ne représente au contraire qu'un mécanisme très particulier valable pour une cellule bien déterminée dans des conditions expérimentales artificielles.

Il est probable que la réalité sera trouvée entre ces extrêmes : il est par exemple vraisemblable que l'expression de formes activées du gène $m y c$ dans des fibroblastes de rongeurs constitue un modèle pour l'étude des nombreux cancers humains où ces gènes sont modifiés. Il est cependant aussi probable que l'état de "haut risque " induit par les gènes du groupe I n'est pas le seul possible. Une indication dans ce sens est fournie par l'étude récente, dans notre laboratoire, des étapes de la transformation induite dans les mêmes cellules de rongeurs par des papillomavirus. Ces virus,dont certains sont trouvés associés à différents cancers humains $[27,28]$, peuvent transformer des fibroblastes de rongeurs en culture. Une étude menée sur le virus du papillome bovin de type I, montre une progression vers un état hautement malin passant par des étapes relativement bien définies $[29,30]$. Comme celles induites par les oncogènes du groupe I, les toutes pre- mières étapes correspondent à des structures cytologiques et à une physiologie proches de la normale, mais ici encore, à un "haut risque ", défini par des fréquences de transformation spontanée trés augmentées et une réactivité vis-àvis des promoteurs chimiques. Ces étapes précoces de la transformation par les papillomavirus sont toutefois clairement différentes de celles induites par les gènes du groupe I : les cellules ne sont pas immortalisées, ni ne deviennent indépendantes des facteurs sériques. L'avenir dira combien d'autres étapes précoces restent encore à découvrir et combien de voies distinctes de cancérisation

\section{Summary}

Stepwise progression of tumoral disease is a phenomenon which has been analyzed in different experimental systems. Studies on the oncogenic polyoma and adenoviruses have allowed the identification of at least two classes of oncogenes sequentially required in the tumoral process. The genes of the first class "immortalize" primary fibroblast cells and reduce their requirement in serum growth factors. Such "activated" cells achieve a complete transformation under the action of the second class genes (either viral or cellular) or after exposure to chemical tumor promoters.

\section{TIRES-A-PART}

François Cuzin : Inserm U 273, Centre de Biochimie de l'Université de Nice, Parc Valrose, o6034 Nice Cedex. 